\begin{tabular}{|c|c|c|c|c|c|}
\hline $\begin{array}{l}\text { Laboratory } \\
\text { number }\end{array}$ & $\begin{array}{c}\text { Published } \\
\text { reference }\end{array}$ & $\begin{array}{l}\text { Original date or } \\
\text { other value }\end{array}$ & $\begin{array}{l}\text { Corrected date } \\
\text { or other value }\end{array}$ & $\partial \mathrm{C}^{14}, \%$ & $\begin{array}{l}\text { A.D./ } \\
\text { B.C. date }\end{array}$ \\
\hline-208 & $170: 239$ & $5400 \pm 300$ & & & 3450 B.C. \\
\hline-209 & $170: 243$ & $1540 \pm 150$ & & & A.D. 410 \\
\hline-210 & $170: 242$ & $5610 \pm 320$ & & & 3660 B.C. \\
\hline-211 & $170: 241$ & $1520 \pm 150$ & & & A.D. 430 \\
\hline-212 & $170: 241$ & Modern & & & \\
\hline-212 bis & $170: 242$ & Modern & & & \\
\hline-216 & $170: 242$ & $20,200 \pm 1000$ & & & 18,250 B.C. \\
\hline-217 & $170: 242$ & $6160 \pm 300$ & & & 4210 B.C. \\
\hline-219 & $170: 238$ & $150 \pm 100$ & & & A.D. 1800 \\
\hline-220 & $170: 239$ & $9500 \pm 400$ & & & 7550 B.C. \\
\hline-221 & $170: 239$ & $7300 \pm 350$ & & & 5350 B.C. \\
\hline-223 & $170: 240$ & $3220 \pm 200$ & & & 1270 B.C. \\
\hline .224 & $170: 240$ & $780 \pm 100$ & & & A.D. 1170 \\
\hline-225 & $170: 240$ & $2440 \pm 160$ & & & 490 B.C. \\
\hline-226 & $170: 238$ & $1700 \pm 150$ & & & A.D. 250 \\
\hline-227 & $170: 237$ & $1010 \pm 120$ & & & A.D. 840 \\
\hline$-228^{13}$ & $170: 236$ & $2440 \pm 180$ & & & 490 B.C. \\
\hline-229 & $170: 243$ & $4030 \pm 250$ & & & 2080 B.C. \\
\hline-230 & $170: 236$ & $4740 \pm 300$ & & & 2790 B.C. \\
\hline-233 & $170: 242$ & $13,600 \pm 450$ & & & 11,650 B.C. \\
\hline-234 & $170: 241$ & $1770 \pm 150$ & & & A.D. 180 \\
\hline-235 & $170: 241$ & $4420 \pm 250$ & & & 2470 B.C. \\
\hline-236 & $170: 241$ & $8240 \pm 350$ & & & 6290 B.C. \\
\hline-237 & $170: 243$ & $5720 \pm 300$ & & & 3770 B.C. \\
\hline-241 & $170: 243$ & $4500 \pm 300$ & & & 2550 B.C. \\
\hline-242 & $170: 239$ & $9200 \pm 400$ & & & 7250 B.C. \\
\hline .243 & $170: 244$ & $8620 \pm 350$ & & & 6670 B.C. \\
\hline .244 & $170: 244$ & $11,520 \pm 400$ & & & 9580 B.C. \\
\hline-245 & $170: 236$ & $1075 \pm 120$ & & & A.D. 875 \\
\hline-247 & $170: 241$ & $7550 \pm 350$ & & & 5600 B.C. \\
\hline
\end{tabular}

${ }^{1}$ Sa (and Gsy, ref. 181) are obsolete designations, two laboratories having joined, beginning 1966, with code designation Gif (ref. 182). Measurements published (refs. 100, 101) before Saclay I, 1964, are included here, but those published after Saclay II, 1965, are not.

${ }^{2}$ Reference standard before 1961 was wood of A.D. 1930 to 1945, not corrected for Suess effect. NBS oxalic-acid standard was adopted in 1961 (ref. 141).

${ }^{3}$ Samples so designated were published, with different sample numbers, in ref. 101.

${ }^{4}$ Published, as sample 0-7, without geologic data, and not republished in Saclay I. Suess-effect correction may not have been applied.

${ }^{5}$ Cf. C-484, from same layer.

${ }^{\circ}$ Also published (sample $\mathrm{T}_{1}, 723 \pm 120$ ) by Gif laboratory, ref. 100

${ }^{7}$ Also published (sample $\mathrm{T}_{2}, 1381 \pm 120$ ) by Gif laboratory, ref. 100.

${ }^{8}$ Also published (sample $B_{1}, 2050 \pm 160$ ) by Gif laboratory, ref. 100; the sample is dated historically as $300 \pm 30$ B.C.

${ }^{9}$ Also published (sample $\mathbf{B}_{2}, 1160 \pm 120$ ) by Gif laboratory, ref. 100.

${ }_{10}$ Also published (sample $\mathrm{B}_{3}, 1271 \pm 120$ ) by Gif laboratory, ref. 100.

${ }^{11}$ Also published (sample $B_{4}, 884 \pm 120$ ) by Gif laboratory, ref. 100.

${ }^{12}$ Also published (sample $B_{5}, 924 \pm 120$ ) by Gif laboratory, ref. 100.

${ }^{13} \mathrm{~A}$ similar sample was published $\left(\mathrm{B}_{7}, 2127 \pm 100\right)$ by Gif laboratory, ref. 100.

${ }^{14} \partial \mathrm{C}^{13}$ values used to compute $\Delta$ are averages of Lamont and Stockholm values.

\title{
Smithsonian Institution, Radiation Biology Laboratory
}

$\begin{array}{cccccc}\begin{array}{c}\text { Laboratory } \\ \text { number }\end{array} & \begin{array}{c}\text { Published } \\ \text { reference }\end{array} & \begin{array}{c}\text { Original date or } \\ \text { other value }\end{array} & \begin{array}{c}\text { Corrected date } \\ \text { or other value }\end{array} & \partial^{14} \mathrm{C}^{14}, \% & \text { A.D./ } \\ \text { SI-3 } 3^{1} & 134: 182 & 10,900 \pm 145 & & & \text { B.C. date } \\ -4^{2} & 134: 182 & 17,145 \pm 210 & & & \text { 8950 B.C. } \\ -4^{2} & 134: 182 & 16,555 \pm 250 & \mathrm{~d} & & 15,195 \text { B.C. } \\ -9^{3} & 134: 182 & 2820 \pm 115 & & & \\ -10^{4} & 134: 182 & 160 \pm 80 & & & 870 \text { B.C. } \\ -12 & 134: 185 & 1030 \pm 60 & \text { See SI-117 d } & \text { A.D. } 1790\end{array}$


Comprehensive Index, 1950-1965

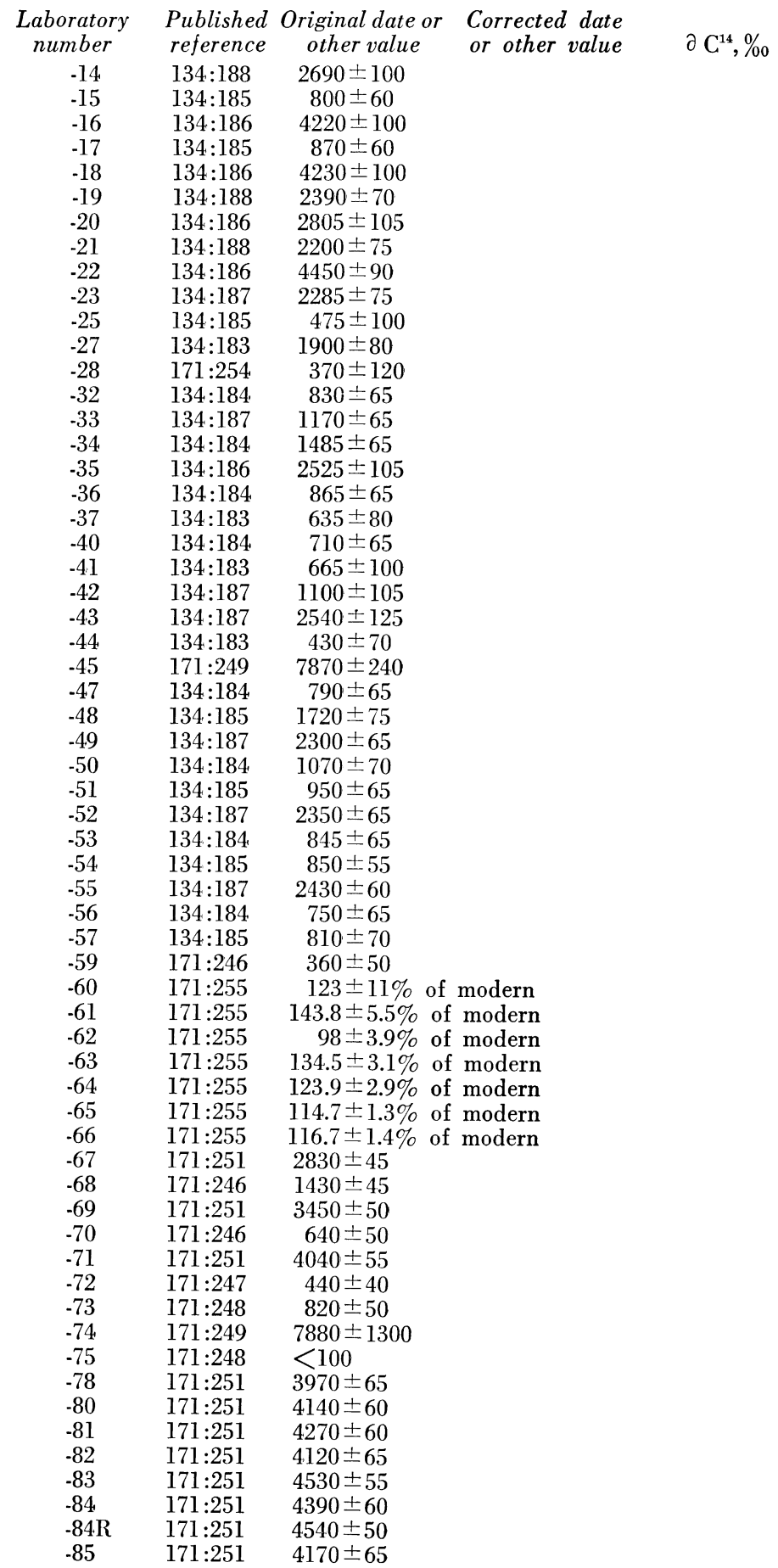

A.D./

B.C. date 740 B.C. A.D. 1150 2270 B.C. A.D. 1080 2280 B.C. 440 B.C.

855 B.C.

250 B.C.

2500 B.C.

335 B.C.

A.D. 1475

A.D. 50

A.D. 1580

A.D. 1120

A.D. 780

A.D. 465

575 B.C.

A.D. 1085

A.D. 1315

A.D. 1240

A.D. 1285

A.D. 850

590 B.C.

A.D. 1520

5920 B.C.

A.D. 1160

A.D. 230

350 B.C.

A.D. 880

A.D. 1000

400 B.C.

A.D. 1105

A.D. 1100

480 B.C.

A.D. 1200

A.D. 1140

A.D. 1590

880 B.C.

A.D. 530

1500 B.C.

A.D. 1310

2090 B.C.

A.D. 1510

A.D. 1130

5930 B.C.

2020 B.C.

2190 B.C.

2320 B.C.

2170 B.C.

2580 B.C.

2440 B.C.

2590 B.C.

2220 B.C. 


\begin{tabular}{|c|c|c|c|c|}
\hline $\begin{array}{l}\text { Laboratory } \\
\text { number }\end{array}$ & $\begin{array}{l}\text { Published } \\
\text { reference }\end{array}$ & $\begin{array}{l}\text { Original date or } \\
\quad \text { other value }\end{array}$ & $\begin{array}{l}\text { Corrected date } \\
\text { or other value }\end{array}$ & $\partial \mathrm{C}^{14}, \%$ \\
\hline-86 & $171: 250$ & $3880 \pm 90$ & & \\
\hline-87 & $171: 247$ & $930 \pm 60$ & & \\
\hline-88 & $171: 247$ & $940 \pm 60$ & & \\
\hline .89 & $171: 247$ & $270 \pm 60$ & & \\
\hline-90 & $171: 253$ & $520 \pm 65$ & & \\
\hline-91 & $171: 253$ & $690 \pm 50$ & & \\
\hline-92 & $171: 253$ & $770 \pm 60$ & & \\
\hline-93 & $171: 253$ & $4580 \pm 60$ & & \\
\hline-95 & $171: 247$ & $270 \pm 50$ & & \\
\hline .97 & $171: 248$ & $290 \pm 60$ & & \\
\hline-98 & $171: 249$ & $8690 \pm 100$ & & \\
\hline-100 & $171: 249$ & $1070 \pm 70$ & & \\
\hline-101 & $171: 249$ & $1050 \pm 70$ & & \\
\hline-102 & $171: 250$ & $1800 \pm 60$ & & \\
\hline-103 & $171: 250$ & $1280 \pm 55$ & & \\
\hline-104 & $171: 248$ & $430 \pm 60$ & & \\
\hline-105 & $171: 248$ & $570 \pm 55$ & & \\
\hline-106 & $171: 248$ & $310 \pm 55$ & & \\
\hline-107 & $171: 252$ & $3320 \pm 170$ & & \\
\hline-108 & $171: 252$ & $2980 \pm 160$ & & \\
\hline-109 & $171: 254$ & $10,110 \pm 400$ & & \\
\hline .110 & $171: 254$ & $510 \pm 60$ & & \\
\hline-111 & $171: 254$ & $1280 \pm 60$ & & \\
\hline-112 & $171: 250$ & $3350 \pm 200$ & & \\
\hline-113 & $171: 253$ & $1440 \pm 50$ & & \\
\hline-114 & $171: 250$ & $6510 \pm 610$ & & \\
\hline-115 & $171: 254$ & $18,940 \pm 1100$ & & \\
\hline-116 & $171: 247$ & $800 \pm 60$ & & \\
\hline$-117^{5}$ & $171: 247$ & $790 \pm 60$ & & \\
\hline-118 & $171: 247$ & $870 \pm 60$ & & \\
\hline-119 & $171: 247$ & $610 \pm 100$ & & \\
\hline-120 & $171: 252$ & $380 \pm 50$ & & \\
\hline-121 & $171: 252$ & $380 \pm 50$ & & \\
\hline-122 & $171: 254$ & $5720 \pm 65$ & & \\
\hline-123 & $171: 254$ & $6950 \pm 400$ & & \\
\hline-124 & $171: 250$ & $2750 \pm 60$ & & \\
\hline-125 & $171: 253$ & $5090 \pm 65$ & & \\
\hline-127 & $171: 245$ & $920 \pm 130$ & & \\
\hline-128 & $171: 245$ & $1300 \pm 140$ & & \\
\hline-129 & $171: 245$ & $350 \pm 200$ & & \\
\hline-130 & $171: 245$ & $620 \pm 120$ & & \\
\hline-131 & $171: 245$ & $740 \pm 130$ & & \\
\hline-135 & $171: 246$ & $240 \pm 120$ & & \\
\hline-136 & $171: 246$ & $310 \pm 120$ & & \\
\hline-137 & $171: 246$ & $360 \pm 120$ & & \\
\hline-138 & $171: 246$ & $910 \pm 120$ & & \\
\hline-139 & $171: 252$ & $310 \pm 50$ & & \\
\hline-140 & $171: 252$ & $760 \pm 50$ & & \\
\hline-141 & $171: 252$ & $800 \pm 50$ & & \\
\hline-142 & $171: 253$ & $730 \pm 50$ & & \\
\hline $\begin{array}{l}{ }^{1} \text { OWU-6. } \\
{ }^{2} \text { OWU-8. } \\
{ }^{3} \text { A-25 bis } \\
{ }^{4} \text { A-298. } \\
{ }^{5} \text { I-581. }\end{array}$ & & & & \\
\hline
\end{tabular}

A.D./

B.C. date

1930 B.C.

A.D. 1020

A.D. 1010

A.D. 1680

A.D. 1430

A.D. 1260

A.D. 1180

2630 B.C.

A.D. 1680

A.D. 1660

6740 B.C.

A.D. 880

A.D. 900

A.D. 150

A.D. 670

A.D. 1520

A.D. 1380

A.D. 1640

1370 B.C.

1030 B.C.

8160 B.C.

A.D. 1440

A.D. 670

1400 B.C.

A.D. 510

4560 B.C.

16,990 B.C.

A.D. 1150

A.D. 1160

A.D. 1080

A.D. 1340

A.D. 1570

A.D. 1570

3770 B.C.

5000 B.C.

800 B.C.

3140 B.C.

A.D. 1030

A.D. 650

A.D. 1600

A.D. 1330

A.D. 1210

A.D. 1710

A.D. 1640

A.D. 1590

A.D. 1040

A.D. 1640

A.D. 1190

A.D. 1150

A.D. 1220 